\title{
Biochemical and molecular assessment of cooking quality and nutritional value of pigmented and non- pigmented whole grain rice
}

\author{
Siriluck Wattanavanitchakorn ${ }^{1}$, Rungtiva Wansuksri ${ }^{2}$, Ekawat Chaichoompu ${ }^{1}$, Wintai \\ Kamolsukyeunyong ${ }^{3}$ and Apichart Vanavichit ${ }^{1,4 *}$
}

\author{
${ }^{1}$ Rice Science Center, Kasetsart University, Kamphaeng Saen Campus, Nakhon Pathom, \\ Thailand \\ ${ }^{2}$ Cassava and Starch Technology Research Unit, National Center for Genetic Engineering \\ and Biotechnology, Bangkok, Thailand \\ ${ }^{3}$ National Center for Genetic Engineering and Biotechnology, PathumThani, Thailand \\ 4Agronomy Department, Faculty of Agriculture Kamphaeng Saen, Kasetsart University, \\ Thailand \\ * Correspondence: Email vanavichit@gmail.com; Tel.: +66-81-527-4070
}

\begin{abstract}
To date, most people prefer softer and stickier rice with high glycaemic index, which has led to the study of the association between the dietary fibre profile and the textural properties to balance between the eating quality, and health benefits. A slight variation was observed in the dietary fibre composition among rice varieties with different amylose content. The percentage of insoluble dietary fibre (IDF) in whole grain rice varied from 1.654.32 while the percentage of soluble dietary fibre (SDF) ranged from 0.26-1.37. We demonstrated that the SDF/IDF ratio was higher in soft texture rice with low amylose and it shared a negative correlation with hardness and chewiness of cooked whole grain rice determined by a texture analyser, which suggested that the distribution of SDF throughout the rice endosperm influences the reduction of the hardness of cooked rice. According to the importance of dietary fibre, we proposed a simple linear regression method to estimate the amount of IDF and total dietary fibre with $r=0.97$ and 0.92 , respectively, of whole grain rice based on its bran layer, determined by the alternative alkaline method. Furthermore, low amylose rice has higher content of $\beta$-glucan and pectin, which are classified as soluble dietary fibre, than high amylose rice. The percentage of $\beta$-glucan and pectin in whole grain rice ranged from 0.03 and 0.07 respectively, for high amylose rice to 0.14 and 0.27 respectively for low amylose rice,
\end{abstract}

Keywords: soft texture rice; dietary fibre prediction; soluble dietary fibre; $\beta$ glucan; pectin; arabinoxylan

\section{Introduction}

Rice (Oryza sativa) is the staple food in almost all Asian countries; however, the evidence regarding the effect of rice consumption on the risk of diabetes mellitus still remains conflicted [1-2]. Therefore, glycaemic index (GI), a value describing the blood glucose response after consumption of foods containing carbohydrate, was originally designed for people with diabetes to serve as a dietary guideline [3-4]. To date, most people prefer softer and stickier rice with high GI, which has led to the study of the association between physicochemical properties, textural properties, cooking properties, nutritional 
properties and starch digestibility to balance between the eating and cooking quality, and health benefits [5-7]. "Dietary fibre", as defined by American Association of Cereal Chemists (AACC), 2000, refers to the edible parts of plant or analogous carbohydrates that are resistant to digestion and absorption by the small intestine in humans with complete or partial fermentation in the large intestine. They are classified into two categories, insoluble-poorly or non-fermentable dietary fibres and soluble-fermentable dietary fibres, which have different health benefits. Insoluble dietary fibres, e.g., cellulose, hemicellulose, arabinoxylan and lignin, contain laxative properties which help increase the faecal bulk and decrease intestinal transit time. This reduces constipation and carcinogen action which leads to colonic cancer. Soluble dietary fibres, e.g., beta-glucan, pectin, gums and mucilages possess gel-forming properties that help increase the transit time through the human digestive tract. This causes delayed gastric emptying, slower glucose absorption and lower plasma cholesterol levels, leading to diminished risk of cardiovascular diseases and type 2 diabetes. Moreover, dietary fibres fermented in the GI tract could provide defence against cancer through the generation of short chain fatty acids [8-10]. In 2008, the definition of dietary fibre was revised again to support nutrition claims and it was decided that "Dietary fibre means carbohydrate polymers with a degree of polymerization not lower than 3" [11]. From this aspect, soluble dietary including high and low molecular weight (HMSDF and LMSDF).

Rice grain is comprised by the hull and edible rice caryopsis, called brown rice. Brown rice or whole grain rice consists of the outer layers, the bran and the germ, and the starchy endosperm. The bran contains the pericarp, seed coat, nucleus and aleurone layer; the starchy endosperm consists of the subaleurone layer and inner endosperm. [12] recently reported the range of total dietary fibre percentage in polished rice, brown rice and rice bran as about 0.7-2.7, 2.9-4.4, and 24-29 respectively. The majority of dietary fibre in cereal grains are derived from the cell wall material [1,13]; [14] recently proposed that the cell wall polysaccharide of polished rice is comprised by $17 \%$ glucans, $19 \%$ xylan, $24 \%$ arabinogalactan and $31 \%$ pectin, which is different from other cereals. Meanwhile, other researchers have reported the profile of non-starch polysaccharide in brown and polished rice to be composed of cellulose, arabinoxylan, pectic substances, fructan, $\beta$-glucan and resistant starch [15-16]; however, the majority composition of rice cell wall has been reported to be cellulose, an insoluble dietary fibre, and pectin, a soluble-fermentable dietary fibre [1]. $\beta$-glucan, a water-soluble dietary fibre, is a major cell wall polysaccharide of oat and barley which is composed entirely of glucose monomers linked together via $\beta-(1 \rightarrow 4)$ and $\beta-(1 \rightarrow 3)$ glycosidic bonds. Pectin is the most complex polysaccharide present in plant cell walls, composed of nearly $70 \%$ galacturonic acid covalently linked at the O-1 and the O-4 position. In terms of the ability to gel, pectin is classified into two types: highmethoxyl pectin (HM pectin), which retains water and forms gel under acid conditions and in the presence of sugar, and low-methoxyl pectin (LM pectin), which forms gel in the presence of divalent cations (i.e., calcium) [17]. Arabinoxylan (AX) is one of the major hemicellulosic component found in cereal grain cell walls and its structure comprises a linear backbone of $\beta-(1 \rightarrow 4)$-linked xylose residues with arabinose substitution at the O-2 and O-3 position. Cereal $\mathrm{AX}$ is classified into 
water-extractable arabinoxylan (WE-AX) and water-unextractable arabinoxylans (WU-AX), which is determined by $\mathrm{Mw}$, the degree and patterns of arabinose substitution and the types of covalent bonding. In cereal grains, most $A X$ are water unextractable (WU-AX), but a smaller part is water extractable (WE-AX). The relationship between nutritional composition, cooking quality, texture and starch digestibility in whole grain rice has so far remained unclear. Thus, we aimed to study the association between dietary fibre profile and the textural properties of whole grain rice samples with different amylose content and gelatinization properties. This could provide useful information to improve high nutrient rice with a softer texture in the future. In this study, we also report on the development of a simple alternative method for the quantitative analysis of dietary fibre in whole grain rice based bran layer.

\section{Materials and methods}

\subsection{Rice varieties}

(Oryza sativa L.): Based on amylose and pigment classification, rice varieties can be grouped into four groups: white high amylose rice PinKaset1 (PK+1), PinKaset+4\#20A09 (PK+4\#20A09), PinKaset4\#78A03 (PK+4\#78A03), Doongara (DGR), Basmati (BMT); white low amylose rice RD 15, RD 43, Sinlek_Kamphaeng (SLK), M7881, Hom Cholasid (HCS); waxy rice Niew Hom Nuan (NHN), RD 6; purple rice Jao Hom Nil (JHN), Klum Hom (KH), Rice Berry (RB); this classification was provided by the Rice Science Center, Kasetsart University, Kamphaeng Saen Campus, Nakhon Pathom, Thailand. Whole grain oat (Avena sativa L.) was purchased from the local market (Bangkok, Thailand).

Bran samples and preparation: Polished rice samples were coarsely ground with a blender followed by fine grinding and screening into particle sizes of $200 \mu \mathrm{m}$ using a speed rotor mill, Pulverisette 14, Fritsch. The flour was stored in an air-tight container at $-20{ }^{\circ} \mathrm{C}$ until required for further analysis. Rice bran samples were separated by two methods: polishing method, by which bran was collected using a rice polisher combined with roller milling; alkaline method: in this method, bran was separated from gelatinized starch after incubation with a $3 \%(\mathrm{w} / \mathrm{v})$ potassium hydroxide $(\mathrm{KOH})$ solution. Before defatting, alkaline treated bran was powdered by grinding with liquid nitrogen. Defatted rice bran was produced using the method given by [18] with modification prior to analysis. Briefly, rice bran was extracted with cold acetone (1:10, w/v) with stirring at $300 \mathrm{rpm}$ for $60 \mathrm{~min}$, followed by centrifugation at $4000 \mathrm{x}$ $\mathrm{g}$ at $4{ }^{\circ} \mathrm{C}$ for $10 \mathrm{~min}$. The supernatant was discarded, and the remaining pellets were re-extracted twice before air-drying under hood overnight. The defatted rice was powdered, sieved as described above and stored in air-tight containers at $-20^{\circ} \mathrm{C}$ until used.

\subsection{Rice bran fraction quantification}

\subsubsection{Polishing method}

In a brief process, bran was removed using a rice polisher followed by roller milling; the bran percentage of whole grain rice was calculated by the weight percentage of bran removed from brown rice as shown in the equation below.

Rice bran (\%, dry basis) $=$ Weight of (unpolished rice-polished rice) $\quad \times 100$

Weight of unpolished rice 


\subsubsection{Alkaline method}

Principally, bran was separated from starch endosperm by incubation with potassium hydroxide $(\mathrm{KOH}), 3 \%(\mathrm{w} / \mathrm{v})$ aqueous solution, which is a versatile substance used for the alkaline degradation test of rice endosperm [19-20]. In a brief process, rice germ samples were separated from brown rice by hand using the process of cutting; the 50 kernels without germ were incubated in $20 \mathrm{ml}$ of $3 \% \mathrm{KOH}$ solution at room temperature. After incubation overnight, rice endosperm starches were gelatinized and separated from rice bran layer; then, detached bran was collected and washed by deionized (DI) water several times. To determine the dry weight of germ and bran layer samples, they were dried in the air oven at $105^{\circ} \mathrm{C}$ for 24 hours and weighed; the percentage of bran weight was calculated using the following equation

$\begin{array}{lll}\text { Rice Germ }(\%, \text { dry basis }) & \frac{\text { Weight of rice germ }}{\text { Weight of whole grain rice }} \times 100 \\ \text { Rice bran layer }(\%, \text { dry basis }) & = & \text { Weight of bran layer } \\ \text { Weight of whole grain rice } & \times 100\end{array}$

Normalization of rice bran: Rice bran was normalized by the surface area based on the oval shape of rice kernel and by the amount of insoluble dietary fibre present in rice bran. The normalized values were calculated using the following equation:

Surface area of $1 \mathrm{~g}$ kernel $\left(\mathrm{cm}^{2}\right)=\underline{\pi \times 1 / 2(\text { Length } \times \text { Width of kernel }(\mathrm{cm})) \times 2 \times 50 \text { kernels }}$

Weight $(\mathrm{g})$ of 50 kernels

Normalized value $=$ rice bran $(\%) \mathrm{x}$ surface area of $1 \mathrm{~g}$ kernel $\left(\mathrm{cm}^{2}\right)$

$=$ rice bran $(\%) \times$ IDF of rice bran $(\%)$

\subsection{Dietary fibre analysis}

\subsubsection{Total dietary fibre (TDF)}

Total dietary fibre (TDF) was determined using the enzymatic method based on the AOAC methods 991.43 and 985.29 (K-TDFR, Megazyme). The sample is subjected to sequential enzymatic digestion by heat stable $\alpha$-amylase, protease and amyloglycosidase. Insoluble dietary fibre (IDF) was quantitated by the gravimetric method while soluble dietary fibre (SDF) was obtained by the liquid chromatography method based on a modification of [21] and AOAC methods 2009.01 and 2011.25 (K-INTDF, Megazyme). All measurements were duplicated. In a brief process, $1 \mathrm{~g}$ of dried and defatted sample (if fat content exceeded $10 \%$ ) was cooked at $\sim 100{ }^{\circ} \mathrm{C}$ for $30 \mathrm{~min}$ with $50 \mathrm{ml}$ of $0.05 \mathrm{M}$ MES-Tris buffer ( $\mathrm{pH}$ 6) and $0.2 \mathrm{ml}$ of thermostable $\alpha$-amylase $(3000 \mathrm{U} / \mathrm{ml})$ to allow gelatinization, hydrolysis and depolymerisation of starch. After cooling, the solution was adjusted to $\mathrm{pH} 7.5$ with $0.275 \mathrm{~N}$ $\mathrm{NaOH}$ and incubated at $60{ }^{\circ} \mathrm{C}$ with $0.1 \mathrm{ml}$ of protease $(50 \mathrm{mg} / \mathrm{ml} ; \sim 350$ tyrosine Units/ml) to solubilise and depolymerise proteins. After $30 \mathrm{~min}$ of incubation, it was adjusted to $\mathrm{pH} 4.5$ with $0.561 \mathrm{~N} \mathrm{HCl}$ and further incubated at $60{ }^{\circ} \mathrm{C}$ for $16 \mathrm{hr}$ with $0.2 \mathrm{ml}$ of amyloglucosidase $(3300 \mathrm{U} / \mathrm{ml})$ in order to hydrolyse starch fragments to glucose. Upon complete digestion, the solution was filtered through fritted crucible (40-100 $\mu \mathrm{m}$ pore size) to separate the insoluble (residue) and soluble (filtrate) fractions. The sediment was washed by $10 \mathrm{ml}$ of $95 \%$ ethanol, $75 \%$ ethanol and acetone, dried and weighed. After washing, the sediment was air oven-dried at $105{ }^{\circ} \mathrm{C}$ overnight and weighed. The weight of the 
precipitate corrected for crude protein and ash formed the total quantity of insoluble dietary fiber (IDF). The filtrate was collected into a $500 \mathrm{~mL}$ Erlenmeyer flask and passed through a column packed with mixed-bed ion-exchange resin. The deionized solution was evaporated to a more concentrated form and filtered through a $0.45 \mu \mathrm{m}$ membrane filter; the filtrate consisting the soluble dietary fiber (SDF) was quantified by highperformance liquid chromatography with a refractive index detector (Shimadzu RID-10A HPLC system, Shimadzu Corporation, Kyoto, Japan). TDF was comprised by the sum of IDF and SDF.

\subsubsection{Beta-glucan}

Beta-glucan was determined in duplicate using enzymatic hydrolysis combined with the colorimetric method (mixed-linkage betaglucan assay kit (K-BGLU, Megazyme) based on AACC method 995.16 and 32-23.01. In this method, lichenase enzyme hydrolyses [(1-3)(1-4)]$\beta$-D-glucan into $\beta$-gluco-oligosaccharides, which is subsequently hydrolysed into D-glucose by $\beta$-glucosidase. The D-glucose produced is assayed using a glucose oxidase/peroxidase reagent. A total of $100 \mathrm{mg}$ of dried and defatted sample (if fat content was more than $10 \%$ ) was hydrated in $0.2 \mathrm{ml}$ of aqueous ethanol $\left(50 \%\right.$, v/v), cooked at $\sim 100{ }^{\circ} \mathrm{C}$ for $3 \mathrm{~min}$ with $4 \mathrm{ml}$ of $20 \mathrm{mM}$ of sodium phosphate buffer, with $\mathrm{pH} 6.5$, then incubated with $10 \mathrm{U}$ of lichenase at $50{ }^{\circ} \mathrm{C}$ for $1 \mathrm{~h}$. The $\mathrm{pH}$ of the solution was adjusted with $5 \mathrm{ml}$ of $200 \mathrm{mM}$ sodium acetate buffer ( $\mathrm{pH} 4)$ before it was centrifuged at $1000 \times \mathrm{g}$ for $10 \mathrm{~min}$. Then, $0.1 \mathrm{ml}$ of the supernatant was incubated with $0.2 \mathrm{U}$ of $\beta$-glucosidase at $50^{\circ} \mathrm{C}$. After 10 min of incubation, the amount of D-glucose released was assayed by a glucose oxidase/peroxidase (GOPOD) reagent kit (K-GLUC, Megazyme, Ireland) through incubation with a GOPOD reagent at $50{ }^{\circ} \mathrm{C}$ for $20 \mathrm{~min}$ followed by measuring the absorbance at $510 \mathrm{~nm}$. $\beta$-glucan content was calculated by multiplying the percentage of D-glucose by the conversion factor $(162 / 180=0.9)$.

\subsubsection{Pectin}

Three fractions of pectin, high methoxyl pectin (HMP), low methoxyl pectin (LMP) and protopectin, can be extracted by three different solutions. The determination of the extracted pectin was performed in duplicate using acid hydrolysis combined with the colorimetric method based on [22]'s method. In a brief process, $1 \mathrm{~g}$ of dried sample was vigorously stirred with $40 \mathrm{ml}$ of DI water for $15 \mathrm{~min}$ and then centrifuged at $1000 \times \mathrm{g}$ for $15 \mathrm{~min}$. The supernatant I containing the HMP fraction was collected into a volumetric flask while the pellet was resuspended with $40 \mathrm{ml} 0.75 \%$ ammonium oxalate solution, which was further stirred for $15 \mathrm{~min}$, and then centrifuged at $1000 \times \mathrm{g}$ for $15 \mathrm{~min}$. The supernatant II containing the LMP fraction was also collected into a volumetric flask, while the last pellet containing protopectin was transferred with DI water into a volumetric flask. Then, $5 \mathrm{ml}$ of $1 \mathrm{~N}$ of $\mathrm{NaOH}$ was added into the flask containing each of these three fractions, allowed to stand for $15 \mathrm{~min}$ and then adjusted the volume to $100 \mathrm{ml}$. The solution was filtered through a Wathman paper no.1 before the filtrate was subjected to colorimetry. A $0.5 \mathrm{ml}$ of the sample solution was hydrolysed by $3 \mathrm{ml}$ of $96 \%$ sulphuric acid at $80{ }^{\circ} \mathrm{C}$ for $8 \mathrm{~min}$. Following the hydrolysis, the amount of D-galacturonic acid released was assayed through incubation with $50 \mu \mathrm{l}$ of $0.15 \%$ solution of 
meta-hydroxy-diphenyl in $0.5 \% \mathrm{w} / \mathrm{w}$ sodium hydroxide at room temperature for $15 \mathrm{~min}$. The absorbance at $520 \mathrm{~nm}$ was measured. The total pectin was the sum of HMP, LMF and protopectin.

\subsubsection{Arabinoxylan}

The level of total- (TAX), water extractable- (WEAX) and water unextractable arabinoxylan (WUAX) in whole grain cereals was determined. WEAX was extracted using the method of [23-24] with modification. A total of $0.25 \mathrm{~g}$ of dried sample was stirred with $2 \mathrm{ml}$ of DI water for $60 \mathrm{~min}$ in a water bath at $40{ }^{\circ} \mathrm{C}$. After stirring, the slurry was centrifuged at $7500 \times \mathrm{g}$ for $20 \mathrm{~min}$, the supernatant was filtered through a Wathman No. 1 filter paper, while the pellet was re-extracted for $10 \mathrm{~min}$ under the same condition. The supernatant from the reextraction was then filtered through filter paper, pooled with the first supernatants and adjusted the volume to $4 \mathrm{ml}$. The arabinoxylan content in the filtrate (WEAX) and the residual (WUAX) was determined by measuring the amount of D-xylose released after the enzymatic hydrolysis of arabinoxylans based on the method of K-xylose, Megazyme. Briefly, both the insoluble and soluble extracts were diluted with DI water to $4 \mathrm{ml}$ total volume in each $20 \mathrm{ml}$ screw-cap culture tube and hydrolysed by adding $1 \mathrm{ml}$ of $6.5 \mathrm{M} \mathrm{HCL}$ (final conc. was $1.3 \mathrm{M}$ $\mathrm{HCl})$. The hydrolysis was accomplished by incubating the tubes in boiling water for $60 \mathrm{~min}$. Following hydrolysis, the sample was neutralized by adding $5 \mathrm{ml}$ of $1.3 \mathrm{M} \mathrm{NaOH}$, quantitatively transferred to a volumetric flask and mixed well. The solution was filtered through Wathman No. 1 prior to the assay. The amount of D-xylose released was determined by incubation of $0.1 \mathrm{ml}$ filtrate with $0.05 \mathrm{ml}$ xylose mutarotase $(\mathrm{XMR}) / \beta$-xylose dehydrogenase $(\mathrm{XMR})$ solution at room temperature for $5 \mathrm{~min}$ followed by the measurement of the absorbance at $340 \mathrm{~nm}$. Basically, the interconversion of the $\alpha$ - and $\beta$-anomeric forms of D-xylose was catalysed by xylose mutarotase. The $\beta$-D-xylose was oxidised by $\mathrm{NAD}^{+}$to D-xylonic acid in the presence of b-xylose dehydrogenase at $\mathrm{pH}$ 7.5. The amount of $\mathrm{NADH}$ is measured by the increase in absorbance at $340 \mathrm{~nm}$. As the arabinoxylan to D-xylose ratio of wheat flour was $62 \%$, this factor was used to measure the arabinoxylan in rice flour samples. The amount of arabinoxylan was calculated by multiplying the percentage of D-xylose by the conversion factor (100/62). Total arabinoxylan $(\%, \mathrm{w} / \mathrm{w})$ was calculated as the sum of water soluble $\mathrm{AX}(\%, \mathrm{w} / \mathrm{w})$ and insoluble $\mathrm{AX}(\%, \mathrm{w} / \mathrm{w})$.

\subsection{Chemical composition analysis}

The amylose content was determined based on the iodinecolorimetric method as per the method of [25]. Total fat was determined using Soxhlet extraction with petroleum ether based on AOAC Method 945.16. The moisture content was measured by the gravimetric method based on the ISO Method 712:2009. Crude protein was determined by Kjeldahl analysis according to AOAC Method 2001.11. Crude ash was determined by incinerating at $525{ }^{\circ} \mathrm{C}$ for $5 \mathrm{~h}$ according to AOAC Method 942.05. All measurements were performed in duplicate.

\subsection{Texture profile analysis}

Texture profile analysis (TPA) of cooked whole grain rice samples based on the method of [26] with modification was conducted with a 
texture analyser (TA-XT plus, Stable Micro System, Godalming, UK). Prior to the texture analysis, whole grain rice samples were soaked in water, with ratio 1:1.8 - 1:2.1: PK+4\#20A09 (1:1.7), PK+4\#78A03 (1:2.1), DGR (1:2.0), RD43 (1:1.5), RD15 (1:1.9), M7881 (1:2.1), SLK (1:2.0) and RB (1:1.8) depending on the water uptake properties in aluminium cups and then cooked in a stainless steel streamer for 30-40 min until no white starch core could be observed. Three warm rice kernels were selected from the middle layer and put onto the base plate of the texture analyser. The cylinder plunger with a $50-\mathrm{mm}$ diameter was set at $30 \mathrm{~mm}$ above the base. A two-cycle compression with $70 \%$ strain, force-versusdistance program was used to allow the probe to travel $30 \mathrm{~mm}$, return and repeat. Pre-test speed, test speed and post-test speed were 10, 5 and $0.5 \mathrm{~mm} / \mathrm{sec}$ respectively, with a contact force of $1 \mathrm{~g}$. The test was repeated on two duplicate samples $(n=3)$, and the parameters recorded from the test curves were hardness, adhesiveness, cohesiveness and springiness. Chewiness is constituted by hardness multiplied by cohesiveness, which is then multiplied by springiness.

\section{Results and Discussion}

3.1. Genetic variation in the dietary fibre characteristic of whole grain rice and rice bran and its effect on cooked whole grain rice's texture

A slight variation in the dietary fibre composition was observed among a series of whole grain rice samples. Table 1 shows that the amount of SDF varied from $0.26 \%$ to $1.37 \%$ and the IDF ranged from $1.65 \%$ to $4.32 \%$, which is relevant to the previous report [27]. The highest SDF value was found in low amylose and waxy rice: RB (1.37\%) and $\mathrm{KH}(1.31 \%)$, and the highest IDF value was observed in waxy rice: RD6 (4.32\%) and $\mathrm{KH}(4.12 \%)$. Moreover, the three highest values of TDF, obtained by calculating the sum of SDF and IDF, were also observed in all waxy rice samples: NHN (4.76\%), RD6 (4.94\%) and KH $(5.43 \%)$. Figure $1 \mathrm{~A}$ shows that rice with lower amylose content has higher dietary fibre content, indicating that the amount of dietary fibre and amylose in whole grain rice shares an inverse correlation. The amount of dietary fibre in rice bran samples, separated from whole grain rice by polishing, was also quantitated; Table 2 indicates that the amount of SDF varied from $1.76 \%$ to $4.47 \%$ and the amount of IDF ranged from $25.25 \%-34.88 \%$, which is in line with the published reports [28-29]. Although the total dietary fibre content in bran was considerably higher than that in whole grain, the major portion of dietary fibre in rice bran was insoluble substance, constituting about $90 \%$ of total dietary fibre, suggesting that only the insoluble type is primarily concentrated in the outer layer of whole grain rice.

When the ratio of SDF and IDF of whole grain rice was compared with the ratio of rice bran, the result revealed that the relative value varied from 0.94 for RD15 to 5.62 for SLK (Table 2, Figure 1). This suggests that there was variation of SDF distribution throughout the endosperm among rice varieties; it was further observed that the hard texture rice with higher amylose exhibited lower relative value while the soft texture rice with lower amylose demonstrated higher relative value. Thus, it is possible that SDF distribution in rice endosperm can influence the texture of whole grain rice. To prove this hypothesis, cooked whole grain rice samples were subjected to texture profile analysis (TPA) conducted using the texture analyser, which mimics the bite of human oral cavity to predict whole grain rice's texture. The 
texture of cooked rice has been described in Table 3. The hardness ranged from $12.74 \mathrm{~N}$ for $\mathrm{RB}$, which had the highest value of SDF/IDF ratio (0.542), to $42.54 \mathrm{~N}$ for $\mathrm{PK}+4 \# 20 \mathrm{~A} 09$, with the SDF/IDF ratio about 0.096; chewiness ranged from 2.04 for $\mathrm{RB}$ to 14.33 for $\mathrm{PK}+4 \# 20 \mathrm{~A} 09$; adhesiveness ranged from $0.016 \mathrm{~N} . \mathrm{sec}$ for PK+4\#20A09 to $0.048 \mathrm{~N}$.sec for RB. Figure 2 depicts that the hardness and chewiness of cooked whole grain rice were negatively correlated with the SDF / IDF ratio of whole grain rice $(\mathrm{r}=-0.89, \mathrm{r}=-0.82$ ) while there was no correlation between the SDF / IDF ratio and adhesiveness (data not shown). The hardness parameter constitutes the force required to bite through rice with molars and chewiness implies the amount of work required to chew the rice, predicting the hardness of rice; meanwhile, adhesiveness is interpreted as the mouthfeel of stickiness [30]. This indicates that the distribution of SDF throughout the rice endosperm influences the reduction of hardness of cooked whole grain rice whereas the amylose content increases the hardness of cooked white rice [26]. In this study, we demonstrated for the first time that the SDF/IDF ratio can be utilised as a predictive indicator of whole grain rice's texture. Additionally, it was observed that pigmented rice, purple low amylose rice (JHN and RB) and purple waxy rice $(\mathrm{KH})$, exhibited a slightly higher percentage of SDF than non-pigmented rice containing the same amylose content: HCS, NHN and RD6 (Table 1). 
Table 1. Chemical compositions of whole grain rice samples.

\begin{tabular}{|c|c|c|c|c|c|c|c|c|}
\hline \multirow{3}{*}{ Varieties } & \multirow{3}{*}{ Colour } & \multirow{3}{*}{$\mathbf{A M}^{a}$} & \multicolumn{6}{|c|}{ Whole grain rice } \\
\hline & & & \multirow{2}{*}{ Amylose } & \multirow[t]{2}{*}{ Starch } & \multicolumn{4}{|c|}{ Dietary fibre $^{b}$} \\
\hline & & & & & SDF & IDF & TDF & SDF/IDF \\
\hline PK4\#20A09 & White & High & $26.86 \pm 0.05$ & $84.29 \pm 0.61$ & $0.26 \pm 0.02$ & $2.70 \pm 0.55$ & 2.97 & 0.096 \\
\hline PK4\#78A03 & White & High & $25.74 \pm 0.67$ & $84.56 \pm 0.99$ & $0.71 \pm 0.01$ & $2.86 \pm 0.25$ & 3.57 & 0.248 \\
\hline Doongara & White & Int & $25.01 \pm 0.29$ & $83.51 \pm 0.74$ & $0.78 \pm 0.16$ & $2.18 \pm 0.84$ & 2.96 & 0.358 \\
\hline Basmati & White & Int & $22.48 \pm 0.51$ & $79.60 \pm 0.48$ & $0.79 \pm 0.12$ & $2.26 \pm 0.25$ & 3.04 & 0.350 \\
\hline RD43 & White & Low & $19.98 \pm 0.40$ & $75.83 \pm 0.97$ & $0.27 \pm 0.02$ & $2.92 \pm 0.03$ & 3.19 & 0.092 \\
\hline RD15 & White & Low & $18.23 \pm 0.09$ & $84.25 \pm 0.28$ & $0.48 \pm 0.00$ & $2.95 \pm 0.30$ & 3.43 & 0.163 \\
\hline PK1 & White & Low & $17.66 \pm 0.30$ & $82.85 \pm 0.23$ & $0.29 \pm 0.07$ & $2.68 \pm 0.33$ & 2.97 & 0.108 \\
\hline M7881 & White & Low & $16.26 \pm 0.12$ & $78.96 \pm 0.68$ & $0.72 \pm 0.24$ & $1.65 \pm 0.24$ & 2.38 & 0.436 \\
\hline SLK & White & Low & $14.86 \pm 0.03$ & $83.80 \pm 1.22$ & $1.05 \pm 0.07$ & $2.18 \pm 0.25$ & 3.22 & 0.482 \\
\hline JHN & Purple & Low & $14.66 \pm 0.04$ & $83.40 \pm 1.98$ & $0.68 \pm 0.25$ & $2.46 \pm 0.02$ & 3.15 & 0.276 \\
\hline HCS & White & Low & $14.42 \pm 0.04$ & $85.80 \pm 0.27$ & $0.25 \pm 0.00$ & $3.67 \pm 0.07$ & 3.91 & 0.068 \\
\hline $\mathrm{RB}$ & Purple & Low & $13.96 \pm 0.03$ & $79.43 \pm 0.27$ & $1.37 \pm 0.39$ & $2.53 \pm 0.14$ & 3.90 & 0.542 \\
\hline NHN & White & Waxy & $7.83 \pm 0.01$ & $84.12 \pm 0.30$ & $1.09 \pm 0.39$ & $3.67 \pm 0.20$ & 4.76 & 0.297 \\
\hline $\mathrm{KH}$ & Purple & Waxy & $6.70 \pm 0.01$ & $76.32 \pm 0.56$ & $1.31 \pm 0.02$ & $4.12 \pm 0.38$ & 5.43 & 0.318 \\
\hline RD6 & White & Waxy & $6.23 \pm 0.02$ & $84.18 \pm 0.37$ & $0.62 \pm 0.01$ & $4.32 \pm 0.06$ & 4.94 & 0.144 \\
\hline
\end{tabular}

Data are expressed as mean \pm standard deviations on dry basis. All compositions are presented as percentages of whole grain flour. ${ }^{a}$ Amylose where High indicates rice with a high amylose content (26-34\%); Int indicates rice with an intermediate amylose content (20-25\%); Low refers to rice with low amylose content (10-19\%); Waxy indicates waxy rice with zero amylose content $(0-9 \%) .{ }^{b}$ Dietary fibre: soluble dietary fibre (SDF), insoluble dietary fibre (IDF), calculated total dietary fibre (TDF = sum of SDF and IDF). 
Table 2. Chemical compositions of rice bran samples.

\begin{tabular}{|c|c|c|c|c|c|c|c|c|c|}
\hline \multirow{3}{*}{ Varieties } & \multicolumn{3}{|c|}{ Rice bran } & \multicolumn{6}{|c|}{ Defatted rice bran } \\
\hline & \multirow{2}{*}{ Colour } & \multirow{2}{*}{$\mathbf{F a t}^{a}$} & \multirow{2}{*}{ Fat } & \multirow[b]{2}{*}{ Starch } & \multicolumn{5}{|c|}{ Dietary fibre content } \\
\hline & & & & & SDF & IDF & TDF & SDF/IDF & Relative $^{b}$ \\
\hline PK4\#20A09 & Brown & $25.02 \pm 0.14$ & $1.39 \pm 0.16$ & $8.55 \pm 1.13$ & $1.99 \pm 0.19$ & $33.80 \pm 0.23$ & 35.79 & 0.059 & 1.64 \\
\hline PK4\#78A03 & Brown & $19.53 \pm 0.03$ & $0.65 \pm 0.00$ & $24.55 \pm 3.06$ & $3.84 \pm 0.24$ & $27.22 \pm 0.76$ & 31.06 & 0.141 & 1.76 \\
\hline RD43 & Brown & $16.42 \pm 1.38$ & $1.13 \pm 0.05$ & $15.91 \pm 0.70$ & $3.18 \pm 0.03$ & $34.17 \pm 1.01$ & 37.35 & 0.093 & 0.99 \\
\hline RD15 & Brown & $19.78 \pm 0.11$ & $1.37 \pm 0.06$ & $20.16 \pm 0.31$ & $4.36 \pm 0.34$ & $25.25 \pm 0.25$ & 29.60 & 0.173 & 0.94 \\
\hline PK1 & Brown & $21.20 \pm 0.24$ & $1.10 \pm 0.11$ & $13.05 \pm 3.44$ & $3.15 \pm 0.19$ & $29.17 \pm 0.06$ & 32.32 & 0.108 & 1.00 \\
\hline M7881 & Brown & $22.48 \pm 0.02$ & $1.05 \pm 0.10$ & $19.24 \pm 5.21$ & $3.58 \pm 0.01$ & $27.75 \pm 0.46$ & 31.33 & 0.129 & 3.38 \\
\hline SLK & Brown & $22.01 \pm 0.31$ & $1.25 \pm 0.03$ & $15.51 \pm 1.26$ & $2.64 \pm 0.24$ & $30.85 \pm 0.22$ & 33.50 & 0.086 & 5.62 \\
\hline JHN & Purple & $21.14 \pm 0.05$ & $0.90 \pm 0.11$ & $16.39 \pm 0.52$ & $4.47 \pm 0.38$ & $29.08 \pm 0.21$ & 33.55 & 0.154 & 1.80 \\
\hline HCS & Brown & $18.91 \pm 0.18$ & $0.83 \pm 0.07$ & $19.68 \pm 3.27$ & $4.44 \pm 0.45$ & $30.47 \pm 0.35$ & 34.91 & 0.146 & 0.47 \\
\hline $\mathrm{RB}$ & Purple & $19.24 \pm 0.03$ & $0.92 \pm 0.03$ & $16.61 \pm 0.39$ & $3.88 \pm 0.99$ & $32.34 \pm 1.23$ & 36.23 & 0.120 & 4.51 \\
\hline NHN & Brown & $20.89 \pm 0.01$ & $1.50 \pm 0.01$ & $11.94 \pm 2.12$ & $1.76 \pm 0.28$ & $29.94 \pm 1.11$ & 31.70 & 0.059 & 5.05 \\
\hline $\mathrm{KH}$ & Purple & $12.98 \pm 0.04$ & $0.39 \pm 0.03$ & $22.67 \pm 3.24$ & $3.59 \pm 0.49$ & $34.88 \pm 0.11$ & 38.47 & 0.103 & 3.09 \\
\hline RD6 & Brown & $19.90 \pm 0.24$ & $1.52 \pm 0.05$ & $15.72 \pm 0.02$ & $3.79 \pm 1.12$ & $30.32 \pm 0.35$ & 34.11 & 0.125 & 1.15 \\
\hline
\end{tabular}

Data are expressed as mean \pm standard deviations on dry basis. All compositions are presented as percentages of defatted rice bran powder except ${ }^{a}$ Fat, which is shown as percentages of rice bran powder. ${ }^{b}$ Relative value is SDF / IDF ratio of whole grain rice flour compared to the ratio of defatted rice bran. 
A

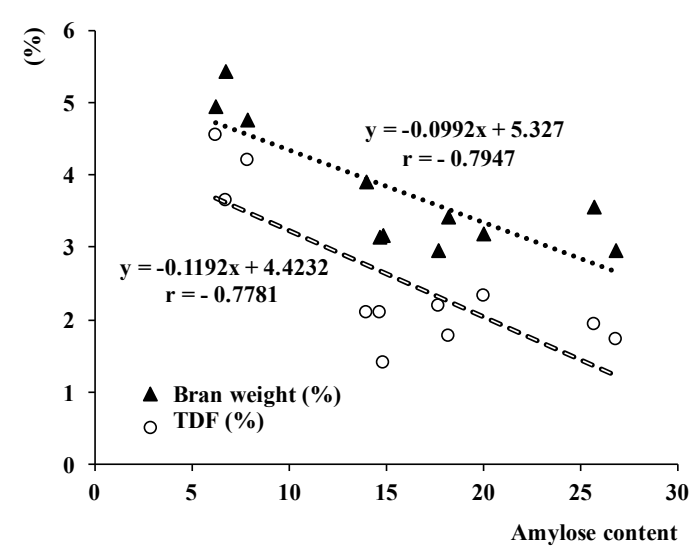

B

Amylose content

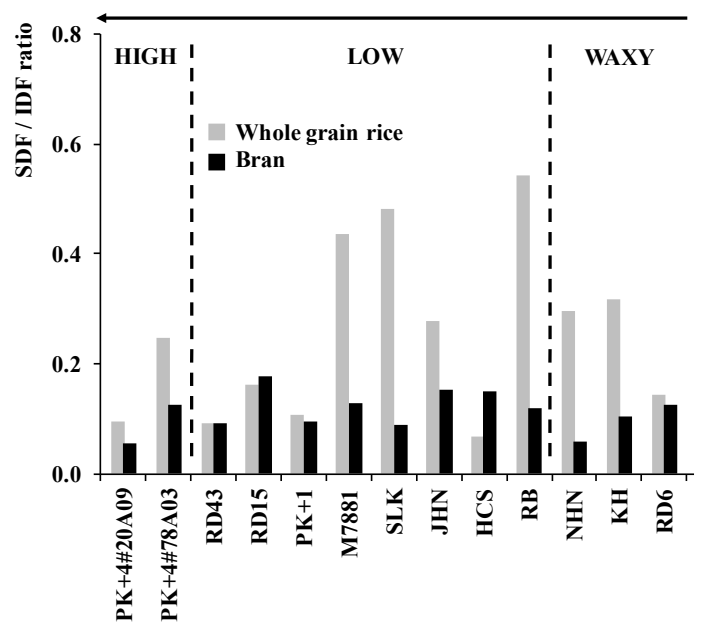

Figure 1. (A) Linear regression between the amylose content of whole grain rice and the percentage of total dietary fibre content or rice bran weight which was determined by the alkaline method; (B) Variation in dietary fibre profile expressed as SDF / IDF ratio of whole grain rice and rice bran samples.

Table 3. Textures of cooked whole grain rice samples.

\begin{tabular}{|c|c|c|c|c|c|}
\hline Varieties & $\begin{array}{c}\text { Hardness } \\
\text { (N) }\end{array}$ & $\begin{array}{c}\text { Adhesiveness } \\
\text { (N.sec) }\end{array}$ & Cohesiveness & Springiness & Chewiness \\
\hline $\mathrm{PK}+4 \# 20 \mathrm{~A} 09$ & $42.54 \pm 0.64$ & $0.016 \pm 0.00$ & $0.48 \pm 0.01$ & $0.69 \pm 0.04$ & $14.33 \pm 0.86$ \\
\hline $\mathrm{PK}+4 \# 78 \mathrm{~A} 03$ & $35.31 \pm 0.59$ & $0.017 \pm 0.01$ & $0.40 \pm 0.01$ & $0.65 \pm 0.01$ & $9.25 \pm 0.30$ \\
\hline DGR & $29.31 \pm 0.85$ & $0.022 \pm 0.01$ & $0.48 \pm 0.01$ & $0.77 \pm 0.01$ & $10.76 \pm 0.75$ \\
\hline RD43 & $24.43 \pm 0.11$ & $0.035 \pm 0.00$ & $0.43 \pm 0.03$ & $0.57 \pm 0.08$ & $6.08 \pm 1.33$ \\
\hline RD15 & $27.80 \pm 0.09$ & $0.023 \pm 0.01$ & $0.42 \pm 0.02$ & $0.60 \pm 0.06$ & $7.12 \pm 1.15$ \\
\hline M7881 & $12.74 \pm 0.33$ & $0.030 \pm 0.01$ & $0.38 \pm 0.01$ & $0.53 \pm 0.02$ & $2.54 \pm 0.02$ \\
\hline SLK & $18.37 \pm 0.03$ & $0.038 \pm 0.02$ & $0.37 \pm 0.01$ & $0.56 \pm 0.01$ & $3.83 \pm 0.11$ \\
\hline $\mathrm{RB}$ & $12.72 \pm 0.11$ & $0.048 \pm 0.02$ & $0.34 \pm 0.04$ & $0.47 \pm 0.04$ & $2.04 \pm 0.40$ \\
\hline
\end{tabular}

A

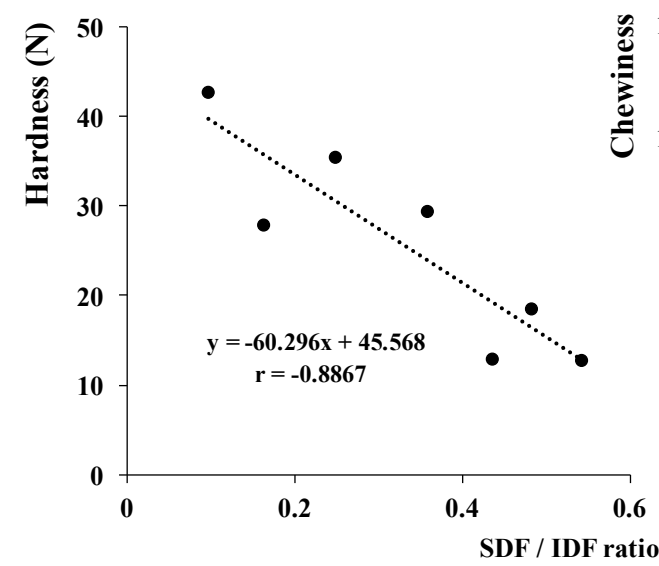

B

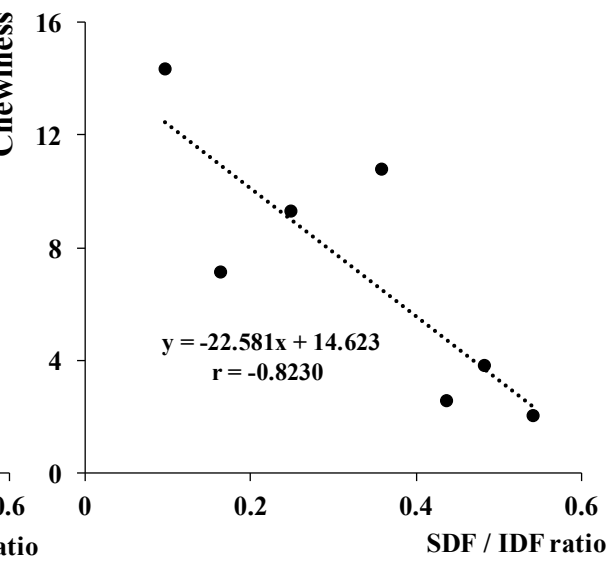

Figure 2. Linear regression between the texture of cooked whole grain rice; (A) Hardness; (B) Chewiness, and the SDF / IDF ratio of whole grain rice. 


\subsection{Simple method for estimation of dietary fibre content in whole grain rice} based on the bran fraction weight

The dietary fibre plays a role in health and it seems to influence the texture of cooked whole grain rice. The importance of dietary fibre led to the investigation of the association between bran weight, determined from both classical polishing method and the alternative method modified from the alkali degradation test, and the dietary fibre of whole grain rice. Table 4 indicates the percentages of germ, bran layer and rice bran, the sum of germ and bran layer, which were estimated by the alkaline method and the polishing method. The results showed that the percentage of alkaline-treated rice bran was 1-2 fold lower than polished rice bran, which is explained by two possible reasons. First is starch contamination in polished rice bran. Second is that some compositions were dissolved in alkaline solution during separation. We further determined the content of crude protein, oil and AX in alkaline treated bran samples compared with milled bran samples; it was found that alkaline treated rice bran had an altered chemical composition, that is, $75.33 \%, 51.22 \%$ and $25.92 \%$ reduction in protein, fat and AX respectively; however, it also showed a $44.67 \%$ increase in total dietary fibre (data not shown).

Figure 3A shows that the percentage of bran layer, determined by the alkaline method, has a strong correlation with the percentage of IDF $(r=0.97)$ and the percentage of TDF $(r=0.92)$. It is not surprising that no relationship was found between the SDF content in whole grain and bran layer - only IDF is mostly localized in the bran layer while SDF is distributed throughout the endosperm, as previously discussed. We further investigated the correlation between rice bran composed of bran layer and germ and the dietary fibre of whole grain rice. Figure 3B shows the strong relationship between rice bran with IDF $(r=0.97)$ but the relationship with TDF became weaker $(r=0.79)$, indicating that the relation between rice bran and dietary fibre in whole grain was slightly weaker compared to bran layer. The possible reason is the difference in chemical compositions between the bran layer and germ, composed of lower dietary fibre but higher protein than bran layer [31-32]. Conversely, no correlation was found between TDF or IDF or SDF of whole grain, and rice bran determined by the polishing method (Figure 3C). Additionally, we hypothesized that kernel size, surface area and dietary fibre content of rice bran could influence the amount of dietary fibre present in whole grain. Thus, the relationship between the normalized value of rice bran and dietary fibre content was also observed. Bran layer value normalized with surface area showed a strong relationship with IDF and TDF ( $\mathrm{r}=0.97$ and 0.91 respectively) as presented in Figure 3D, while the normalized value of rice bran showed a strong relationship with IDF $(r=0.96)$ and with TDF $(r=0.80)$ (Figure $3 E)$. Although Figure 3F indicates a weak correlation with IDF $(r=0.67)$, no correlation was found between the normalized value of rice bran determined by the polishing method and TDF. Lastly, rice bran was also normalized by the amount of insoluble dietary fibre in rice bran; Figure 3G showed that only the bran layer, determined by the alkaline method, showed a strong relationship with IDF and TDF $(r=0.96$ and 0.92 respectively). Thus, it was concluded that the amount of IDF and TDF was simply estimated by the liner regression model, $\%$ IDF $=0.6643 \mathrm{X}+$ $1.3482, \% \mathrm{TDF}=0.7598 \mathrm{X}+1.8764$, where $\mathrm{X}$ represents the percentage of bran layer determined by the alkaline method as shown in Figure 4A. 
We also demonstrated the predicted value of IDF, and TDF was strongly correlated with the experimental value of IDF and TDF quantitated by the AOAC standard method (Figure 4B).

Rice bran, composed of pericarp, seed coat, aleuronic layer and germ, is rich in non-starch constituents: total dietary fibre $(24 \%-29 \%)$, crude fat $(15 \%-19.7 \%)$, crude protein $(11.3 \%-14.9 \%)$ and crude ash $(6.6 \%-9.9 \%)$ [27]. Interestingly, rice bran also consisted of starchy endosperm, which is derived from the outer layer of brown rice with high variation in available carbohydrate, 34\%-62\%; [33,27] and [34] revealed that there is a variation in the milling characteristics among rice varieties due to the size and thickness of the kernel. They also suggested that cultivars with higher ratio of length and width (L:W ratio) show higher degree of milling (DOM). Besides the varietal differences, the type of mill used for milling also highly affects the bran percentage and milling recovery [35-36]. In the present study, the weight of rice bran separated by the polishing method was expressed as DOM. Unsurprisingly, we also found an inverse correlation between the amount of starch and fat in rice bran samples (Table 2), suggesting that there was a difference in the degree of starch contamination from the outer layer of brown rice among rice bran samples. Due to the measurement error of the polishing method, we proposed the alternative method modified from the alkali degradation test to provide a more accurate value of the rice bran, which was further used for prediction of rice dietary fibre.

The alkali degradation test, also referred to as the alkaline spreading method, has been extensively employed in rice quality studies as an indirect estimation method of the gelatinization temperature and the cooking quality [19,37-38]. As the difference in alkaline resistant properties of rice varieties and rice grain were fully gelatinized by the $\mathrm{KOH}$ concentration, ranging from $0.6-2.3 \%$ [20], the $\mathrm{KOH}$ solution with $3 \%$ concentration was used for complete starch gelatinization to separate rice bran from starchy endosperm. During alkali spreading, $\mathrm{KOH}$ gelatinizes starch, particularly its amorphous region, causing degradation of the long linear and branched chains of amylose and amylopectin; this results in rice grain gelatinization [38]. Although rice bran, which is mainly composed of fibre, lipid and protein, can be separated from the starchy endosperm fraction becoming starch gel, some of the constituents, e.g., oil, protein and arabinoxylan (AX), were dissolved in an alkaline solution during separation [39-40]. The alkaline solvent also solubilized AX from the cell wall matrix through the disruption of hydrogen and covalent bonds, resulting in loss of alkali-solubilized AX during the washing of rice bran; [41-42] and [43] have also demonstrated that oil and protein are mostly concentrated in the outer portion of the rice bran layer while the highest rice bran saccharide is found in the inner portion of the bran layer. 
Table 4. Comparison of rice bran weight as determined by the alkaline method and polishing method, shape, weight and surface area of whole grain rice samples.

\begin{tabular}{|c|c|c|c|c|c|c|c|c|c|c|c|}
\hline \multirow[b]{2}{*}{ Varieties } & \multicolumn{3}{|c|}{ Alkaline $^{a}$} & polishing $^{b}$ & \multicolumn{4}{|c|}{ Shape } & \multirow[b]{2}{*}{$\begin{array}{l}50 \text { kernel } \\
\text { weight }(\mathrm{g})\end{array}$} & \multicolumn{2}{|c|}{ Surface area ${ }^{c}$} \\
\hline & $\begin{array}{l}\text { Germ } \\
(\%)\end{array}$ & $\begin{array}{c}\text { Bran layer } \\
(\%)\end{array}$ & $\begin{array}{c}\text { Rice bran } \\
(\%)\end{array}$ & $\begin{array}{c}\text { Rice bran } \\
(\%)\end{array}$ & $\begin{array}{l}\text { Length } \\
(\mathrm{cm})\end{array}$ & $\begin{array}{l}\text { Width } \\
\text { (cm) }\end{array}$ & $\begin{array}{l}\text { Thickness } \\
(\mathrm{cm})\end{array}$ & $\begin{array}{l}\mathrm{L}: \mathrm{W} \\
\text { ratio }\end{array}$ & & $\begin{array}{l}50 \text { kernels } \\
\left(\mathrm{cm}^{2}\right)\end{array}$ & $\begin{array}{c}1 \mathrm{~g} \text { kernel } \\
\left(\mathrm{cm}^{2}\right)\end{array}$ \\
\hline PK4\#20A09 & $3.11 \pm 0.2$ & $71.70 \pm 0.10$ & 4.81 & 8.81 & $0.71 \pm 0.04$ & $0.23 \pm 0.01$ & $0.18 \pm 0.00$ & 3.09 & $1.0897 \pm 0.02$ & $12.93 \pm 0.98$ & $11.87 \pm 0.90$ \\
\hline PK4\#78A03 & $3.35 \pm 0.0$ & $12.09 \pm 0.11$ & 5.44 & 8.94 & $0.74 \pm 0.03$ & $0.20 \pm 0.00$ & $0.16 \pm 0.01$ & 3.70 & $0.9779=$ & 11.6 & $11.91 \pm 0.56$ \\
\hline RD43 & $3.46 \pm 0.2$ & $32.32 \pm 0.04$ & 5.78 & 9.55 & $0.75 \pm 0.02$ & $0.20 \pm 0.00$ & $0.17 \pm 0.01$ & 3.75 & $0.9943 \pm 0.01$ & $11.83 \pm 0.33$ & $11.90 \pm 0.33$ \\
\hline RD15 & $3.20 \pm 0.2$ & $22.21 \pm 0.34$ & 5.41 & 12.91 & $0.75 \pm 0.02$ & $0.20 \pm 0.01$ & $0.16 \pm 0.01$ & 3.75 & $0.9254 \pm 0.01$ & $11.63 \pm 0.59$ & $12.57 \pm 0.64$ \\
\hline PK1 & $2.96 \pm 0.0$ & $02.02 \pm 0.14$ & 4.98 & 8.42 & $0.76 \pm 0.04$ & $0.21 \pm 0.01$ & $0.17 \pm 0.01$ & 3.62 & $1.0696=$ & $12.61 \pm$ & $11.79 \pm 1.15$ \\
\hline M7881 & $3.76 \pm 0.2$ & $43.07 \pm 0.70$ & 6.83 & 9.97 & $0.79 \pm 0.03$ & $0.19 \pm 0.01$ & $0.16 \pm 0.01$ & 4.16 & $0.9559 \pm 0.01$ & $11.85 \pm 0.89$ & $12.40 \pm 0.93$ \\
\hline SLK & $2.40 \pm 0.3$ & $01.33 \pm 0.04$ & 3.73 & 9.00 & $0.69 \pm 0.01$ & $0.20 \pm 0.01$ & $0.17 \pm 0.01$ & 3.45 & $0.9780 \pm 0.02$ & $11.00 \pm 0.56$ & $11.25 \pm 0.57$ \\
\hline $\mathrm{JHN}$ & $2.50 \pm 0.1$ & $32.02 \pm 0.08$ & 4.52 & 10.73 & $0.67 \pm 0.02$ & $0.19 \pm 0.01$ & $0.16 \pm 0.02$ & 3.53 & $0.8181 \pm 0.00$ & $9.86 \pm 0.03$ & $12.05 \pm 0.03$ \\
\hline $\mathrm{RB}$ & $1.87 \pm 0.1$ & $12.16 \pm 0.05$ & 4.03 & 11.19 & $0.68 \pm 0.03$ & $0.18 \pm 0.01$ & $0.15 \pm 0.01$ & 3.78 & $0.7706 \pm 0.00$ & $9.33 \pm 0.01$ & $12.11 \pm 0.01$ \\
\hline NHN & $2.33 \pm 0.2$ & $63.78 \pm 0.42$ & 6.11 & 9.29 & $0.90 \pm 0.0$ & $0.22 \pm 0.01$ & $0.19 \pm 0.01$ & 4.09 & $1.3128 \pm 0.02$ & $15.28 \pm 0.52$ & $11.64 \pm 0.40$ \\
\hline $\mathrm{KH}$ & $3.67 \pm 0$. & $93.80 \pm 0.16$ & 7.47 & 13.51 & $0.84 \pm 0.0$ & $0.21 \pm 0.01$ & $0.18 \pm 0.01$ & 4.00 & $1.0456 \pm 0.03$ & $13.67 \pm 1.11$ & $13.07 \pm 1.06$ \\
\hline RD6 & $3.49 \pm 0.2$ & $94.52 \pm 0.01$ & 8.01 & 9.27 & $0.71 \pm 0.03$ & $0.23 \pm 0.01$ & $0.16 \pm 0.00$ & 3.09 & $0.9455 \pm 0.02$ & $12.58 \pm 0.14$ & $13.31 \pm 0.15$ \\
\hline
\end{tabular}

Data are expressed as mean \pm standard deviations on dry basis. ${ }^{a}$ Alkaline method: germ and bran layer are presented as percentages of the dry weight of whole grain rice while rice bran is the sum of germ and bran layer. ${ }^{b}$ Polishing method: rice bran was presented as the degree of milling, weight of bran percentage removed from brown rice. cSurface area was calculated based on the calculation of oval shape surface as explained in materials and methods where surface area of 1 $\mathrm{g}$ kernel was surface area of 50 kernels divided by the dry weight of 50 kernels. 

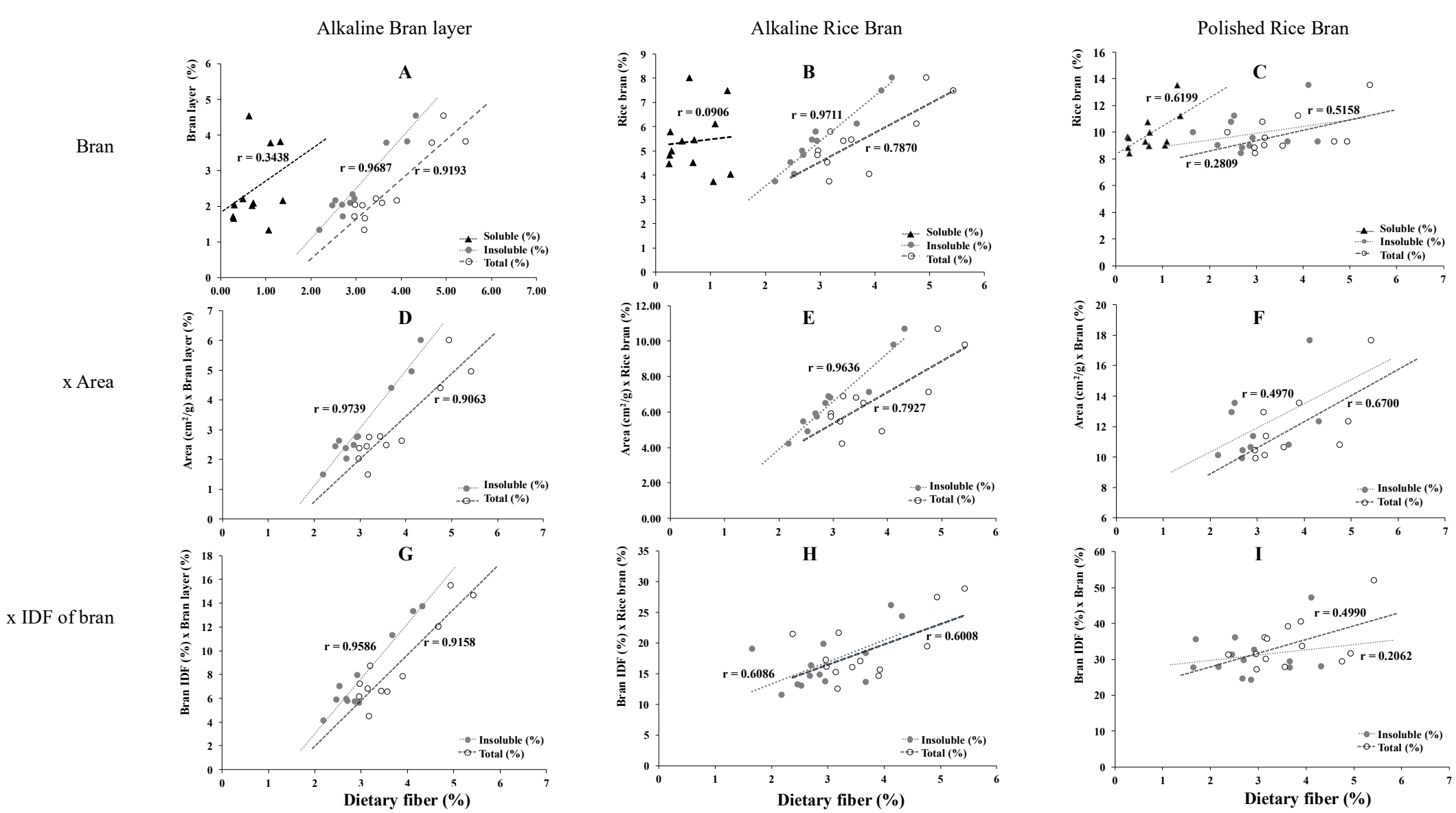

Figure 3. (A-C) Linear regression between the dietary fibre content: SDF, IDF and TDF, and bran layer (A) or rice bran (B), as determined by alkaline method, or rice bran (C), as determined by polishing method; (D-F) Linear regression between the dietary fibre content and surface area multiplied by bran layer (D) or rice bran (E), as determined by alkaline method, or rice bran (F), as determined by polishing method; (G-I) Linear regression between the dietary fibre content and IDF content of rice bran multiplied by bran layer (D) or rice bran (E), as determined by alkaline method, or rice bran (F), as determined by polishing method. 
A

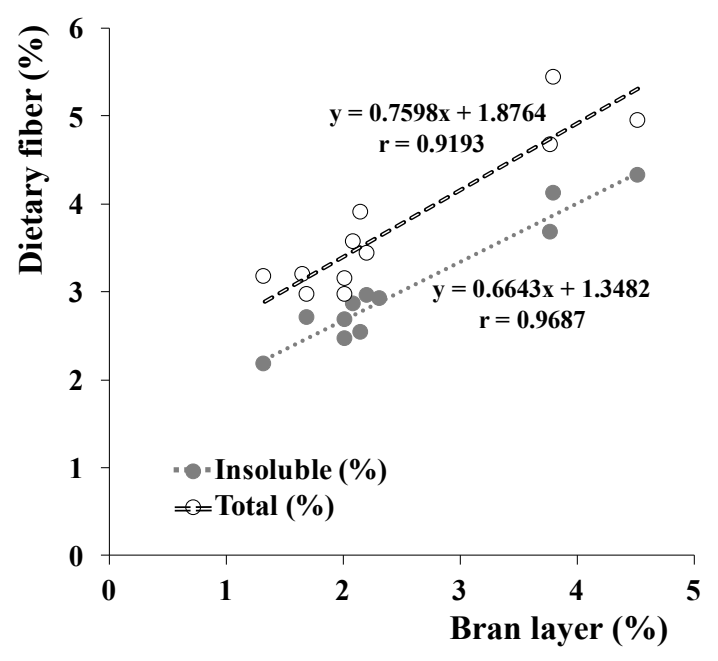

B

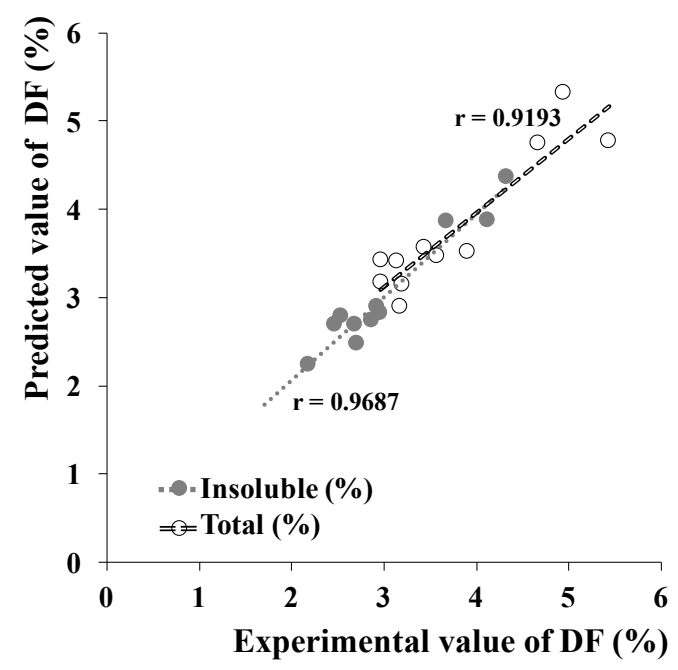

Figure 4. (A) Linear regression between the dietary fibre content: IDF and TDF and the bran layer determined by alkaline method; (B) Linear regression between the IDF and TDF, predicted value from the alkaline method and the estimated value from standard method.

\subsection{Higher content of $\beta$-glucan and pectin in low amylose and waxy rice}

Till now, limited information was available on the composition of soluble dietary fibre in whole grain pigmented and non-pigmented rice with differing amylose content and in their bran samples. We investigated the composition of soluble dietary fibre including $\beta$-glucan, pectin and soluble arabinoxylan in whole grain and rice bran samples. Table 5 shows that the amount of $\beta$-glucan in whole grain rice varied from $0.03 \%$ to $0.14 \%$. The highest amount of $\beta$-glucan, found in low amylose RB rice, was 4.7-fold higher than the lowest amount, observed in high amylose rice, PK+4\#20A09 and PK+478A03. In case of bran, $\beta$ glucan content varied from $0.21 \%$ to $0.40 \%$ and the highest value, also found in RB rice bran, was only 1.9-fold higher than the lowest value, obtained from M7881 rice bran. This suggests that there was greater variation in $\beta$-glucan content of whole grain rice than rice bran. A decrease in amylose content related to increased $\beta$-glucan content in whole grain rice with a strong correlation $(r=0.83)$, while there was no correlation between the content of $\beta$-glucan in rice bran and amylose content in whole grain rice (Figure 5). Moreover, $\beta$-glucan content in bran was about 2-7 fold higher than that in whole grain rice, and the highest relative value was found in high amylose rice while the lowest relative value was observed in waxy rice, consistent with the result of SDF as discussed above. This is explained by two possible reasons: greater distribution of $\beta$-glucan throughout the starchy endosperm in waxy- and low-amylose rice; difference in the ratio of endosperm and bran in which dietary fibre is concentrated among rice cultivars. We also determined the amount of $\beta$-glucan in oat grain and bran as a known sample control, and the results revealed that oat bran contains about $5.99 \% \beta$-glucan, 1.45 -fold higher than oat grain in relation to the reported values [44-48]. 
Table 5. Comparison of the $\beta$-glucan and pectin content in whole grain and defatted rice bran.

\begin{tabular}{ccccc}
\hline \multirow{2}{*}{ Varieties } & \multicolumn{2}{c}{$\beta$-glucan } & & Pectin \\
\cline { 2 - 5 } & Grain $^{a}$ & Bran $^{b}$ & Grain & Bran \\
\hline PK4\#20A09 & $0.03 \pm 0.00$ & $0.24 \pm 0.01$ & $0.07 \pm 0.01$ & $0.26 \pm 0.02$ \\
PK4\#78A03 & $0.03 \pm 0.00$ & $0.25 \pm 0.00$ & $0.10 \pm 0.01$ & $0.27 \pm 0.01$ \\
RD43 & $0.03 \pm 0.00$ & $0.24 \pm 0.01$ & $0.16 \pm 0.04$ & $0.31 \pm 0.02$ \\
RD15 & $0.06 \pm 0.03$ & $0.29 \pm 0.00$ & $0.25 \pm 0.00$ & $0.29 \pm 0.03$ \\
PK1 & $0.07 \pm 0.02$ & $0.22 \pm 0.00$ & $0.11 \pm 0.00$ & $0.30 \pm 0.05$ \\
M7881 & $0.08 \pm 0.00$ & $0.21 \pm 0.00$ & $0.14 \pm 0.06$ & $0.29 \pm 0.08$ \\
SLK & $0.11 \pm 0.00$ & $0.26 \pm 0.00$ & $0.16 \pm 0.02$ & $0.33 \pm 0.06$ \\
JHN & $0.11 \pm 0.00$ & $0.26 \pm 0.03$ & $0.27 \pm 0.02$ & $0.32 \pm 0.01$ \\
HCS & $0.09 \pm 0.01$ & $0.23 \pm 0.01$ & $0.25 \pm 0.05$ & $0.27 \pm 0.02$ \\
RB & $0.14 \pm 0.03$ & $0.40 \pm 0.04$ & $0.19 \pm 0.00$ & $0.42 \pm 0.03$ \\
NHN & $0.10 \pm 0.04$ & $0.33 \pm 0.01$ & $0.21 \pm 0.03$ & $0.32 \pm 0.03$ \\
KH & $0.10 \pm 0.01$ & $0.23 \pm 0.03$ & $0.26 \pm 0.02$ & $0.39 \pm 0.07$ \\
RD6 & $0.06 \pm 0.00$ & $0.27 \pm 0.02$ & $0.29 \pm 0.04$ \\
\hline
\end{tabular}

Data are expressed as mean \pm standard deviations on dry basis. $\beta$-glucan and pectin are presented as percentages of ${ }^{a}$ whole grain rice flour or ${ }^{b}$ defatted rice bran powder.

Table 6. Water-extractable (WEAX) and Water-unextractable (WUAX) arabinoxylan of selected whole grains rice varieties.

\begin{tabular}{cccc}
\hline \multirow{2}{*}{ Varieties } & \multicolumn{2}{c}{ Arabinoxylan } & Total AX $^{\boldsymbol{c}}$ \\
\cline { 2 - 4 } & WEAX $^{\boldsymbol{a}}$ & WUAX $^{\boldsymbol{b}}$ & 0.82 \\
PK4\#20A09 & Whole grain rice & 0.82 \\
PK4\#78A03 & $0.04 \pm 0.01$ & $0.78 \pm 0.01$ & 0.76 \\
RD43 & $0.03 \pm 0.01$ & $0.78 \pm 0.01$ & 0.89 \\
SLK & $0.03 \pm 0.00$ & $0.73 \pm 0.02$ & 0.94 \\
RB & $0.03 \pm 0.00$ & $0.86 \pm 0.01$ & 0.95 \\
NHN & $0.03 \pm 0.02$ & $0.91 \pm 0.00$ & 0.89 \\
SLK & $0.03 \pm 0.01$ & $0.92 \pm 0.02$ & 0.72 \\
RD6 & $0.03 \pm 0.00$ & $0.86 \pm 0.01$ & \\
PK4\#20A09 & $0.03 \pm 0.02$ & $0.69 \pm 0.00$ & \\
RB & Defatted rice bran & 11.42 \\
NHN & $0.19 \pm 0.01$ & $8.03 \pm 0.21$ & 8.26 \\
\hline Data are & $0.21 \pm 0.03$ & $8.91 \pm 0.06$ & 9.11 \\
\hline
\end{tabular}

Data are expressed as mean \pm standard deviations on dry basis. Arabonoxylan (AX) is presented as percentages of whole grain rice flour or defatted rice bran powder composed of ${ }^{a}$ water extractable AX (WEAX), ${ }^{b}$ water unextractable AX (WUAX) and total AX. 

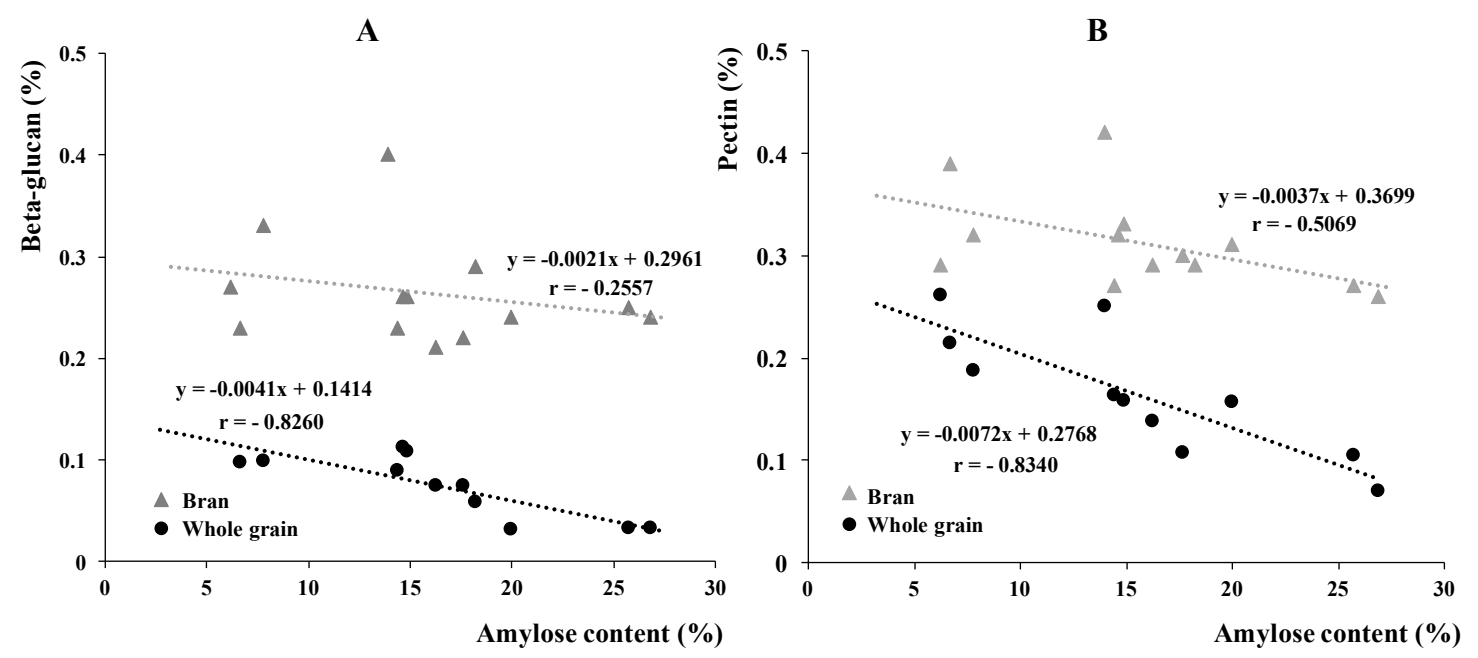

Figure 5. Linear regression between the amylose content of whole grain rice and (A) the total $\beta$-glucan content; (B) the total pectin of either whole grain rice or defatted rice bran.

A previous study has reported that whole grain rice contains about $0.24 \%$ total pectin [22]; however, there is no information regarding pectin content in whole grain rice with different amylose content. Table 5 shows that the percentage of pectin in whole grain rice varied from $0.07 \%-0.27 \%$ and the highest pectin content was found in low-amylose JHN rice and waxy RD6 rice, was 3.8-fold higher than the lowest content, which was found in high-amylose rice, PK+4\#20A09. In case of bran, the amount of pectin varied from $0.26 \%-0.42 \%$ and the highest value, also found in RB rice bran, was only 1.6-fold higher than the lowest value obtained from $\mathrm{PK}+4 \# 20 \mathrm{~A} 09$ rice bran. A decline in amylose content related to an increased pectin content in whole grain rice with a strong correlation $(\mathrm{r}=0.83)$, while no correlation was found between the content of pectin in rice bran and amylose content in whole grain rice (Figure 5). Total pectin content in bran was about 1-4 fold higher than that in whole grain rice and the highest relative value was found in high amylose rice while the lowest relative value was observed in waxy rice, consistent with the result of SDF and $\beta$-glucan as previously discussed. This suggests that the proportion of pectin in endosperm is almost similar to that in the rice bran and the pectin distribution in the endosperm of waxy- and low-amylose rice is higher than that of high amylose rice. Table 6 also shows that whole grain rice contained water unextractable AX (WUAX) in the range $0.69 \%-0.92 \%$ while there was no difference in the water extractable AX (WEAX), $0.03 \%$. This suggests that only $3 \%-4 \%$ is water-soluble type but most of arabinoxylan in whole grain rice are water-insoluble type. In case of bran, the amount of WUAX varied from $8.03 \%-11.23 \%$ and the amount of WEAX varied from $0.19 \%-0.21 \%$. WUAX and WEAX content in bran was about $10-14$ fold and 6-7 fold respectively, higher than that in whole grain rice.

$\beta$-glucan, a water-soluble dietary fibre, is a major cell wall polysaccharide composed entirely of glucose monomers linked together via $\beta-(1 \rightarrow 4)$ and $\beta-(1 \rightarrow 3)$ glycosidic bonds. [49] reported that $\beta$-glucan is a minor component in the endosperm cell wall material and the aleurone layer of rice grain. Although $\beta$-glucan in whole grain rice and rice bran was found in a small amount, a few studies have demonstrated the functional properties including structure, antioxidant 
and prebiotic properties of beta-glucan extracts of rice bran compared to oat beta-glucan [50-51]. The US Food and Drug Administration allows a health claim that a daily intake of $3 \mathrm{~g}$ of soluble oat $\beta$-glucan can lower the risk of coronary heart disease by reducing low-density lipoprotein cholesterol [52-53]. $\beta$-glucan plays a beneficial role against cardiovascular disease, dyslipidemia, insulin resistance and obesity due to its fermentability and viscous properties [54]. Moreover, $\beta$-glucan can directly enhance the immune system via interaction with immune cells [55].

Pectin is a family of covalently linked galacturonic acid-rich plant cell wall polysaccharides and it is the most complex polysaccharide in plant cell walls including in rice. [56] uncovered the proportion of pectic substances in cell wall materials including seed coat (7\%), aleurone layer $(11 \%)$, germ $(23 \%)$ and endosperm $(27 \%)$. Although there is no information available regarding the health benefits of pectin in rice, several reports revealed that pectin has multiple positive effects on human health via reduction of post-prandial glycemic responses and maintenance of normal blood cholesterol concentration owing to its viscosity property [14]. The consumption of at least $6 \mathrm{~g} /$ day of pectin is necessary to have a significant effect in cholesterol reduction [57].

Soluble arabinoxylan is claimed to have beneficial effects on human health through the selective promotion of certain bacterial species of the gut flora, which is known as the prebiotic effect, and reducing the postprandial blood glucose level [58-60]. Although WEAX in whole grain rice and rice bran was found in little amounts, [61] reported that glycoproteins, arabinoxylan- and arabinogalactan-hydroxyproline were found in water soluble polysaccharides extracted from the rice endosperm and rice bran. They also demonstrated that glycoprotein can modulate the human complement activity. The human complement system or immunological defence system plays an important role in the host's defence against microbial pathogens. Conversely, the aberrant activation of this system may contribute to inflammatory and degenerative disease.

\section{Conclusion}

A slight variation in dietary fibre composition of whole grain rice was observed among rice varieties containing different amylose content; the amount of SDF in whole grain rice was found to influence the texture of cooked rice. This study demonstrated that the ratio of SDF and IDF of whole grain rice has an inverse correlation with the hardness of cooked whole grain rice. Furthermore, the study proposed a simple method to estimate the amount of insoluble dietary fibre and total dietary fibre of whole grain rice based on its bran layer, which was determined by the alternative alkaline method. Interestingly, softtexture rice, low amylose and waxy rice contained higher soluble dietary fibre, $\beta$-glucan, and pectin content than rice with harder texture, high amylose. The results suggested that high soluble dietary fibre affects the softness and can serve the future trend of breeding rice for health and well-being.

Author Contributions: S.W., R.W., and A.V. designed this research; S.W. and R.W. performed the experiments; S.W. and A.V. wrote the manuscript. All authors have read and agreed to the published version of the manuscript. 
Funding: This project was supported by NSRF via the Program Management Unit for Human Resources and Institutional Development, Research, and Innovation (Grant No. B16F630088). M.P. was supported in part by a Graduate Program Scholarship from Kasetsart University.

Data Availability Statement: The data presented in this study are available on request from the corresponding author.

Conflicts of Interest: The authors declare no conflict of interest.

\section{References}

1. Butardo, V.M.; Sreenivasulu, N. Tailoring grain storage reserves for a healthier rice diet and its comparative status with other cereals. Int. Rev. Cell. Mol. Bio. 2016, 323, 31-70.

2. Rohman, A.; Helmiyati, S.; Hapsari, M.; Setyaningrum, D.L. Rice in health and nutrition: Mini review. Int. Food. Res. J. 2014, 21, 13-24.

3. Venn, B.J.; Green, T.J. Glycemic index and glycemic load: Measurement issues and their effect on dietdisease relationships. Eur. J. Clin. Nutr. 2007, 51, S122-S131.

4. Kaur, B.; Ranawana, V.; Henry, J. The glycemic index of rice and rice products: A review, and table of GI values. Crit. Rev. Food. Sci. Nutr. 2016, 56, 215-236.

5. Khatun, A.; Waters, D.L.E.; Liu, L. A review of rice starch digestibility: Effect of composition and heat-moisture processing. Starch. 2019, 1900090.

6. $\quad$ Pang, Y.; Ali, J.; Wang, X.; Franje, N.J.; Revilleza, J.E.; Xu, J.; Li, Z. Relationship of rice grain amylose, gelatinization temperature and pasting properties for breeding better eating and cooking quality of rice varieties. 2016, PLOS One. https://doi.org/10.1371/journal.pone.0168483

7. Lu, S.; Cik, T.; Lii, C.; Lai, P.; Chen, H. Effect of amylose content on structure, texture and-amylose reactivity of cooked rice. Food. Sci. Tech. 2013, 54, 224-228.

8. Smith, C.E.; Tucker, K.L. Health benefits of cereal fibre: a review of clinical trials. Nutr Res Rev. 2011, 24, $118-131$.

9. Papandreou, D.; Noor, Z.T.; Rashed, M. The role of soluble, insoluble fibers and their bioactive compounds in cancer: A mini review. Food Nutri. Sci. 2015, 6, 1-11. doi: 10.4236/fns.2015.61001

10. Mudgil, D. The interaction between insoluble and soluble fiber. Dietary fiber for the prevention of cardiovascular disease ( $1^{\text {st }}$ Ed). 2017, Academic Press. 35-59.

11. McCleary, B.V. Development of an integrated total dietary fiber method consistent with the codex alimentarius definition. Cereal. Foods. World. 2010, 55, 24-28.

12. Juliano, B.O. The cereal grains rice: Overview. Encyclopedia of Food Grains, 2016, 1, 125-129.

13. Englyst, H.N.; Quigley, M.E.; Hudson, G.J. Dietary fiber analysis as non-starch polysaccharides. Encyclopedia of Analytical Chemistry, 2006. https://doi.org/10.1002 /9780470027318.a1006

14. Lovergrove, A.; Kosik, O.; Bandonill, E.; Abilgos-Ramos, R.; Romero, M.; Sreenivasulu, N.; Shewry, P. Improving rice dietary fiber content and composition for human health. J. Nutr. Sci. Vitaminol. 2019, 65, S48-S50.

15. Lai, V.M.F.; Lu, S.; He, W.H.; Chen, H.H. Non-starch polysaccharide compositions of rice grains with respect to rice variety and degree of milling. Food. Chem. 2006, 101, 1205-1210.

16. Dodevska, M.S.; Djordjevic, B.I.; Sobajic, S.S.; Miletic, I.D.; Djordjevic, P.B.; Dimitrijevic-Sreckovic, V.S. Characterisation of dietary fibre components in cereals and legumes used in Serbian diet cereals and legumes used in Serbian diet. Food. Chem. 2013, 141, 1624-1629.

17. BeMiller, J. Pectins. Carbohydrate chemistry for food scientists, $3^{\text {rd }}$ ed.; BeMiller, J. Eds.; Workhead Publishing and AACC International Press, UK, 2018; pp. 303-312. https://doi.org/10.1016/B978-0-12-8120699.00015-7

18. Ren, X.; Zhao, X.; Turcotte, F.; Deschênes, J.S.; Tremblay, R.; Jolicoeur, M. Current lipid extraction methods are significantly enhanced adding a water treatment step in Chlorella protothecoides. Microb. Cell. Fact. 2017, 16, 26.

19. Little, R.R.; Hilder, G.B.; Dawson, E.H. Differential effect of dilute alkali on 25 varieties of milled white rice. Cereal. Chem. 1958, 35, 111-126.

20. Bhattacharya, K.R.; Sowbhagya, C.M. An improved alkali reaction test for rice quality. J. Food. Technol. 1972, 7, 323-331.

21. Ohkuma, K.; Matsuda, I.; Katta, Y. New method for determining total dietary fiber by liquid chromatography. J. AOAC. Int. 2000, 83, 1013-1019.

22. Bailoni, L.; Schiavon, S.; Pagnin, G.; Tagliapietraa, F.; Bonsembiante, M. Quanti-qualitative evaluation of pectins in the dietary fibre of 24 foods. Ital. J. Anim. Sci. 2005, 4, 49-58. https://doi.org/10.4081/ijas.2005.49.

23. Fleury, M.D.; Edney, M.J.; Campbell, L.D.; Crow, G.H. Total, water-soluble and acid-soluble arabinoxylans in western Canadian barleys. Can. J. Plant. Sci. 1997, 77, 191-196.

24. Delcour, J.A.; Win, H.V.; Grobet, P.J. Distribution and structural variation of arabinoxylans 
common wheat mill streams. J. Agri. Food. Chem. 1999, 47, 271-275.

25. Juliano, B.O. A simplified assay for milled-rice amylose. Cereal. Sci. Today. 1971, 16, 335-340.

26. Yu, S.; Ma, Y.; Sun, D. Impact of amylose content on starch retrogradation and texture of cooked milled rice during storage. J. Cereal. Sci. 2009, 50, 139-144.

27. Juliano, B.O. The cereal grains rice: Overview. Encyclopedia. of. Food Grains, 2016, 1, 125-129.

28. Hamid, A.A.; Luan, Y.S. Functional properties of dietary fiber from defatted rice bran. Food. Chem. 2000, 68, 15-19. DOI: 10.1016/S0308-8146(99)00145-4

29. Daou, C; Zhang, H. Functional and physiological properties of total, soluble, and insoluble dietary fibres derived from defatted rice bran. J. Food. Sci. Technol. 2014, 51, 3878-3885. DOI 10.1007/s13197-0130925-y

30. Lyon, B.G.; Champagne, E.T.; Vinyard, B.T.; Windham, W.R. Sensory and instrumental relationships of texture of cooked rice from selected cultivars and postharvest handling practices. Cereal. Chem. 2000, 77, 64-69.

31. Moongngarm, A.; Daomukda, N.; Khumpika, S. Chemical compositions, phytochemicals, and antioxidant capacity of rice bran, rice bran layer, and rice germ. APCBEE. Procedis. 2012, 2, 73-79.

32. Rondanelli, M.; Miccono, A.; Peroni, G.; Nichetti, M.; Infantino, V.; Spadaccini, D.; Alalwan, T.A.; Faliva, M.A.; Perna, S. Rice germ macro- and micronutrients: A new opportunity for the nutraceutics. Nat. Prod. Res. 2019. doi: 10.1080/14786419.2019.1660329

33. Shibuya, N.; Iwasaki, T. Structural features of rice bran hemicelluloses. Phytochemistry, 1984, 24, 285-289.

34. Gujral, H.S.; Singh, J.; Sodhi, N.S.; Singh, N. Effect of milling variables on the degree of milling of unparboiled and parboiled rice. Int. J. Food. Prop. 2002, 5, 193-204.

35. Pokhrel, A.; Dhakal, A.; Sharma, S; Poudel, A. Evaluation of physicochemical and cooking characteristics of rice (Oryza sativa L.) Landraces of Lamjung and Tanahun districts, Nepal. Int. J. Food. Sci. 2020, Article ID 1589150. https://doi.org/10.1155/2020/ 1589150

36. Din, S.U., Bhattacharya, K.R. On the meaning of the degree of milling of rice. J. Food. Technol. 1978, 13, 99-105.

37. Mariotti, M.; Fongaro, L.; Catenacci, F. Alkali spreading value and Image Analysis. J. Cereal. Sci. 2010, 52, 227-235.

38. Tuaño, A.P.P.; Ricafort, C.H.; del Rosario, E.J. Estimation of alkali spreading value and gelatinization temperature of some Philippine rice varieties using digital photometr. Philipp. Agric. Scientist. 2018, 101, 4, 354-362

39. Lim, S.T.; Lee, J.H.; Shin, D.H.; Lim, H.S. Comparison of protein extraction solutions for rice starch, isolation and effects of residual protein content on starch pasting properties. Starch. 1999, 4, 120-125.

40. Phongthai, S.; Homthawornchoo, W.; Rawdkuen, S. Preparation, properties and application of rice bran protein: A review. Int. Food. Res. J. 2017, 24, 25-34.

41. Zhang, H.; Wu, F.; Xu, D.; Xu, X. Endogenous alpha-amylase explains the different pasting and rheological properties of wet and dry milled glutinous rice flour. Food. hydrocoll. 2021, 113, 106425.

42. Bender, D.; Nemeth, R.; Wimmer, M.; Gotschhofer, S.; Biolchi, M.; Torok, K.; Tomoskozi, S.; D'Amico, S.; Schoenlechner, R. Optimization of arabinoxylan isolation from rye bran by adapting extraction solvent and use of enzymes. J. Food. Sci. 2017, 82, 2562-2568.

43. Schramm, R.; Abadie, A.; Hua, N.; Xu, Z.; Lima, M. Fractionation of the rice bran layer and quantification of vitamin E, oryzanol, protein, and rice bran saccharide. J. Biol. Eng. 2007, $1,9$. doi:10.1186/1754-1611-1-9

44. Demirbas, A. B-glucan and mineral nutrient contents of cereals grown in Turkey. Food. Chem. 2005, 90, 773-777.

45. Flander, L.; Salmenkallio-Marttila, M.; Suortti, T.; Autio, K. Optimization of ingredients and baking process for improved wholemeal oat bread quality. LWT - Food Sci. Technol, 2007, 40, 860-870.

46. Knudsen, K.E.B. Fiber and non starch polysaccharide contents and variation in common crops used in broiler diets. Poult. Sci. 2014, 93, 2380-2393.

47. Izydorczyk, M.S.; McMilan, T.; Bazin, S.; Kletke, J.; Dushnicky, L.; Dexter, J.; Chepuma, A.; Rossnagel, B. Milling of Cannadian oats and barley for functional food ingredients: Oat bran and barley fibrerich fractions. Can. J. Plant. Sci. 2014, 94, 573-586.

48. Esteban, R.M.; Molla, E.; Benitez, V. Source of fiber. Dietary fiber for the prevention of cardiovascular disease ( $1^{\text {st }}$ Ed). 2017, Academic Press. 121-146.

49. Shibuya, N. Comparative studies on the cell wall polymers obtained from different parts of rice grains. 1989, American Chemical Society, Washington, DC. 333-344.

50. Phuwadoipaisarn, P. The influence of conditions on beta-glucan extraction from Thai rice bran cultivars and their biological properties. Proceeding of $95^{\text {th }}$ The IIER International Conference, Osaka, Japan, $8^{\text {th }}-9^{\text {th }}$ February 2017. ISBN: 978-93-86083-34-0

51. Neethi, R.P.; Anie, Y. Extraction of water soluble beta-glucan from rice bran. Sch. Acad. J. Biosci. 2017, 5, 766-770.

52. US Food and Drug Administration. Health claims meeting significant scientific agreement (SSA). 
http://www.fda.gov/Food/IngredientsPackagingLabeling/ Labeling Nutrition/ucm20068 76.htm. Updated October 5, 2015. Accessed November 17, 2015.

53. Rebello, C.J.; O’Neil, C.E.; Greenway, F.L. Dietary fiber and satiety: The effects of oats and satiety. Nutr. Rev. 2016, 74, 131-147.

54. El Khoury, D.; Cuda, C.; Luhovyy, B.L.; Anderson, G.H. Beta glucan: Health benefits in obesity and metabolic syndrome. J. Nutr. Metab. 2012, 851362. doi:10.1155/2012/851362

55. Slavin, J. Fiber and prebiotics: Mechanisms and health benefits. Nutrients. 2013, 5, 1417-1435. doi:10.3390/nu5041417

56. Shibuya, N.; Nakane, R.; Yasui, A.; Tanaka, K.; Iwasaki, T. Comparative studies on cell wall preparations from rice bran, germ and endosperm. Cereal Chem. 1985, 62, 252-258.

57. Ginter, E.; Kubec, F.J.; Vozar, J.; Bobek, P. Natural hypocholesterolemic agent: Pectin plus ascorbic acid. Int. J. Vitic. Nat. Resour. 1979. 49, 406-408.

58. Kiszonas, A.M.; Courtin, C.M.; Morris, C.F. A critical assessment of the quantification of wheat grain arabinoxylans using a phloroglucinol colorimetric assay. Cereal. Chem. 2012, 89, 143-150.

59. Liu, Z.; Rochfort, S. A simple method for simultaneous quantification of total arabinoxylans and fructans in wheat flour. J. Agric. Food. Chem. 2014, 62, 8319-8324. https://doi.org/10.1016/j.foodhyd.2016.10.016

60. Huang, V.T.; Perdon, A.A. Major changes in cereal biopolymers during ready-to-eat-cereal processing. Breakfast cereals and how they are made, $3^{\text {rd }}$ ed.; Perdon, A.; Schonauer, S.; Poutanen, K., Eds.; Workhead Publishing and AACC International Press, UK, 2020; pp. 109-140. https://doi.org/10.1016/B978-0-12-8120439.00006-0.

61. Yamagishi, T.; Yamaguchi, A; Saito, T.; Seguchi, M. Complement-modulating water-soluble polysaccharide fractions in two global staple cereals: Rice and wheat. J. Sci. Food. Agri. 2008, 88, 805-812. 